\title{
GRAND: a database of gene regulatory network models across human conditions
}

\author{
Marouen Ben Guebila ${ }^{\circledR 1, \dagger}$, Camila M. Lopes-Ramos ${ }^{1, \dagger}$, Deborah Weighill ${ }^{\circledR 1}$, \\ Abhijeet Rajendra Sonawane ${ }^{2}$, Rebekka Burkholz', Behrouz Shamsaei ${ }^{3}$, John Platig ${ }^{4}$, \\ Kimberly Glass ${ }^{1,4}$, Marieke L. Kuijjer ${ }^{\circledR 5,6}$ and John Quackenbush ${ }^{\circledR 1,4,{ }^{*}}$ \\ ${ }^{1}$ Department of Biostatistics, Harvard School of Public Health, Boston, MA, USA, ${ }^{2}$ Center for Interdisciplinary \\ Cardiovascular Sciences, Cardiovascular Medicine, Brigham and Women's Hospital, Harvard Medical School, \\ Boston, MA 02115, USA, ${ }^{3}$ Division of Biostatistics and Bioinformatics, Department of Environmental and Public \\ Health Sciences, University of Cincinnati College of Medicine, Cincinnati, OH, USA, ${ }^{4}$ Channing Division of Network \\ Medicine, Department of Medicine, Harvard Medical School and Brigham and Women's Hospital, Boston, MA, USA, \\ ${ }^{5}$ Center for Molecular Medicine Norway, Faculty of Medicine, University of Oslo, Oslo, Norway and ${ }^{6}$ Leiden University \\ Medical Center, Leiden, The Netherlands
}

Received June 01, 2021; Revised August 17, 2021; Editorial Decision August 23, 2021; Accepted September 08, 2021

\section{ABSTRACT}

Gene regulation plays a fundamental role in shaping tissue identity, function, and response to perturbation. Regulatory processes are controlled by complex networks of interacting elements, including transcription factors, miRNAs and their target genes. The structure of these networks helps to determine phenotypes and can ultimately influence the development of disease or response to therapy. We developed GRAND (https://grand.networkmedicine. org) as a database for computationally-inferred, context-specific gene regulatory network models that can be compared between biological states, or used to predict which drugs produce changes in regulatory network structure. The database includes 12 468 genome-scale networks covering 36 human tissues, 28 cancers, 1378 unperturbed cell lines, as well as 173013 TF and gene targeting scores for 2858 small molecule-induced cell line perturbation paired with phenotypic information. GRAND allows the networks to be queried using phenotypic information and visualized using a variety of interactive tools. In addition, it includes a web application that matches disease states to potentially therapeutic small molecule drugs using regulatory network properties.

\section{GRAPHICAL ABSTRACT}
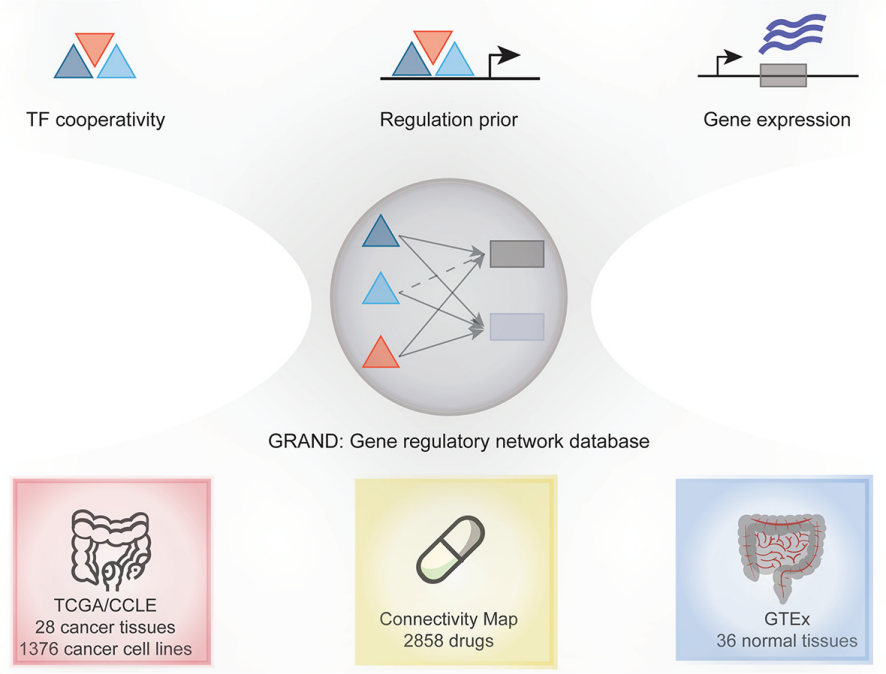

\section{INTRODUCTION}

Gene expression is controlled by complex networks of interacting factors within the cell that help define cellular, tissue and organismal phenotypes, and that allow cells to respond to external and internal perturbations. Dysregulation of these regulatory processes can lead to disease, including cancer $(1,2)$. Although multiple factors play a role in gene regulation $(3,4)$, the most common regulators are transcription factors (TFs) and microRNAs (miRNAs). miRNAs are small non-coding RNAs involved in mRNA

\footnotetext{
*To whom correspondence should be addressed. Tel: +1 617432 9028; Fax: +1 617432 5619; Email: johnq@hsph.harvard.edu

${ }^{\dagger}$ The authors wish it to be known that, in their opinion, the first two authors should be regarded as Joint First Authors.

Present address: Deborah Weighill, UNC Lineberger Comprehensive Cancer Center, University of North Carolina at Chapel Hill, Chapel Hill, NC, USA. 
post-transcriptional regulation. In most cases, miRNAs bind to short complementary sequences within the $3^{\prime}$ untranslated regions of mRNAs, causing mRNA degradation or translational repression, and thereby silencing their target mRNA $(2,5)$. TFs bind to TF-specific motif sequences in the promoter regions of their target genes and modulate gene expression by interacting or interfering with other key transcriptional proteins including RNA polymerase $(4,6)$. Several experimental techniques such as ChIP-seq (7) and ChEC-seq (8) allow measurement of the binding of TFs across the genome, providing evidence of regulatory associations. However, such experiments typically only look at small numbers of transcription factors and are not scalable to population level studies.

Because large-scale experimental determination of context-specific regulatory processes has proven challenging, there is a growing recognition of the need for methods to infer gene regulatory networks (GRNs) and for comparing regulatory network architectures between phenotypes or experimental groups. The rapidly growing volume of genomic and transcriptomic data in human health (9) and disease (10) has greatly facilitated the development of GRN inference methods using bulk tissue data (11-16) and single-cell data (17-23) and has provided the validation data necessary to refine and tune these methods. Similarly, the availability of data sets that include both transcriptional profiling and phenotypic response to perturbagens, including small molecule drugs (24-26), provide opportunities to study how expression and regulatory network structures correlate with phenotype. Several web resources were developed recently to provide users with online inference tools and databases of computationally-predicted context-specific networks (27-30). For example, iNetModels (31) has a catalog of coexpression networks in normal and cancer tissues as well as integrated multi-omic networks. ChEA (32) aggregates several sources of evidence to infer upstream TF regulators of a given gene list. Additional examples include TargetScan (33) that predicts miRNA targets and GIANT (30) that predicts tissue-specific networks for a gene of interest using Bayesian integration over a large set of data sources to generate hypotheses about functional associations. Finally, GRNdb (27) provides a set of regulatory networks predicted by SCENIC (17) using bulk and single-cell data, however, the lack of interactive visualization as well as the lack of availability of the source code of network inference and analysis pipeline could challenge community engagement and reproducibility. The above-mentioned resources were built using approaches that require several gene expression samples to infer context-specific, aggregate GRNs across all samples. However, none of them consider sample-specific GRNs to account for essential differences in phenotypic variation between patients such as sex, age, and ethnicity. In particular, there is a lack of GRN modeling in the Cancer Cell Line Encyclopedia (CCLE) database (34), which provides gene expression samples for more than 1376 cell lines with a single gene expression sample for each cell line. In this case, aggregate methods fail to compute GRNs for individual CCLE cell lines because they require several samples.
Since 2013, our research group has developed and validated a collection of GRN inference tools designed to work with various input data (35-39). This family of tools is collectively referred to as the 'Network Zoo' (netzoo; netzoo.github.io). The baseline method in netzoo, PANDA (35), is derived from the understanding that TFs can interact with their target genes to activate or repress the expression of those genes. It also recognizes that some TFs exert their influence as part of multi-TF complexes and that genes that are regulated by the same TFs are likely to exhibit similar patterns of expression. Consequently, PANDA takes as input (i) an initial regulatory network based on mapping TFs to their potential target genes in the genome based on TF binding motifs, as well as (ii) protein-protein interaction (PPI) data and (iii) the gene co-expression relationships across the samples being studied. PANDA then uses message passing (35) to iteratively search for agreement between these data sources until it arrives at an optimal network structure. This conceptual framework is flexible in that other sources of regulatory information and constraints can be introduced. For example, PUMA (36) extends PANDA by including miRNAs as regulators of expression, while LIONESS (37) uses a linear interpolation approach to extract single-sample networks for each research subject (or biological sample) in a study population. OTTER (38) estimates a gene regulatory network by optimizing graph matching between three networks derived from the three input datasets. DRAGON (39) builds a multi-omic network using a variation of Gaussian Graphical Models (GGMs) by implementing covariance shrinkage to estimate partial correlations.

We previously used the netzoo methods, particularly PANDA and LIONESS, to infer tens of thousands of GRN models. We analyzed these networks in a number of published studies, including GRN comparison of 36 'normal' tissues and two cell lines from the Genotype Tissue Expression (GTEx) project $(36,40,41)$ and six cancers from The Cancer Genome Atlas (TCGA) (38,42-44). Although each study included detailed descriptions of the data and methods used to generate these networks, there was no appropriate data repository for publishing, querying, and visualizing the GRN models themselves due to the large number of genome-scale networks with millions of edges that required more than $6 \mathrm{~TB}$ of data storage. Given that the inference of these networks took thousands of computational hours, we recognized that the lack of an appropriate network repository to host thousands of network models created substantial obstacles to the reuse of our published network models to investigate additional questions.

To address the need for such a resource and to facilitate the query and analysis of these networks, we created the Gene Regulatory Network Database (GRAND; https: //grand.networkmedicine.org). GRAND catalogs curated networks created using netzoo tools together with samplespecific phenotypic information. To supplement the existing collection of networks, and to allow comparison of health and disease phenotypes with perturbations arising from treatment with small molecule candidate therapeutic compounds, we generated additional $173013 \mathrm{TF}$ and gene targeting scores, corresponding to the weighted outdegree for TFs and weighted indegree for genes (44). These scores were derived from network models of cell lines treated with 
2858 small molecule compounds cataloged by the Connectivity Map (24) project, 1376 cell line networks from the CCLE database (34) accounting for TF and miRNA regulation, and 22 cancer types from TCGA. In total, GRAND contains 12468 GRNs representing samples from 36 human tissues, 28 cancer types, 1378 cell lines, and 2,858 small molecule screening assays. The majority of these networks model cis-transcriptional regulation at the TF level, and a subset of networks model post-transcriptional regulation using miRNA information. Our goal is to continue to grow both the number and diversity of network types in GRAND as the field of GRN inference evolves and to add new analytical tools as more phenotypes and experimental samples become publicly available.

\section{DATA COLLECTION AND DATABASE CONTENT}

\section{Overview of network models in GRAND}

GRNs in GRAND are built on the conceptual framework first presented in PANDA in which we model GRNs explicitly as the interaction between TFs and their target genes (Figure 1A). GRAND includes additional network inference tools to model the regulation between miRNAs and their target genes (PUMA), to build single-sample GRNs (LIONESS), to construct GRNs using relaxed graph matching (OTTER), and to use Gaussian Graphical Models to build multi-omic networks (DRAGON). Our starting point in assembling GRAND was the collection of network models we had previously constructed using data from GTEx, TCGA, and GEO $(36,38,40,41,45)$ (Figure 1B and $\mathrm{C}$ ). To these, we added network models inferred using data available from the Connectivity Map (CMAP) project (24) and CCLE (34). The CMAP project measured gene expression in human cell lines after exposure to a combination of 2858 approved and investigational drugs and additional chemical compounds. The CCLE collected multiomic data - miRNA and gene expression, methylation, histone marks, and protein levels - for $>1000$ cell lines (Supplementary Table S1). These networks can be selected using phenotypic information (Supplementary Figure S1) and visualized on the browser using a dedicated module (Figure 2).

\section{GENE REGULATORY NETWORKS}

\section{Small molecule resource}

The Connectivity Map phase I (24) and phase II (26) amassed gene expression profiles for human cell lines exposed to various drugs and drug candidates; we selected 2858 that were cataloged in the Drug Repurposing Hub (DRH) (46). The DRH has essential information on compounds that includes drug indication, chemical structure, and targets. This provided 173013 gene expression profiles (level 4) for drug exposure across normal and cancer cell lines, doses, and sampling times that were used for GRNs reconstruction (Supplementary Figure S2).

The Connectivity Map directly profiles the expression of 1000 genes (the L1000 genes) and uses these data to infer the expression levels of the remaining genes. For network inference, we used the complete set of 12328 sequenced and inferred genes (https://grand.networkmedicine.org/genes/), also referred to as All Inferred Genes (AIG) set. For these data, we used GPU-accelerated MATLAB implementations of PANDA and LIONESS in the netzoo package (netZooM v 0.5.1) (47) to infer sample-specific GRNs for each of the 173013 profiles, and subsequently computed TF and gene targeting scores for each network.

\section{Cancer resource}

The cancer resource in GRAND includes both aggregate networks and patient-specific networks across 28 cancer types. In total, 2811 patient-specific networks were generated for colon cancer, pancreatic cancer and glioblastoma. The colon GRNs were derived using expression data from 445 samples in TCGA and 1193 samples found in GEO as described previously (42) (Supplementary Figure S3). Glioblastoma networks were generated from 953 samples in TCGA and 70 samples from the German Glioma Network (GGN) (43). Pancreatic cancer networks were generated from 150 samples from TCGA spanning both basallike and classical subtypes (44).

We used PANDA to generate aggregate networks for 22 cancer types in TCGA, and OTTER to generate networks for three cancer types (breast, liver, and cervical cancer) in TCGA (38) that were used to validate the accuracy of this new inference tool (38). The validation of these specific networks using ChIP-seq data from ReMap (7) as described by Weighill et al. (38) was added in the 'Network Benchmarking' section.

\section{Tissue resource}

The tissue resource made use of GTEx data to construct TF and miRNA GRNs for 36 'normal' human tissues (Supplementary Figure S3). We used PANDA to build the aggregate TF networks (41), and PUMA to build the aggregate miRNA networks (36). Using PANDA and LIONESS, we also built 8279 sample-specific TF networks (37).

\section{Cell line resource}

The cell line resource includes TF and miRNA aggregate networks built using PANDA (32) and PUMA (36), respectively, for LCLs and fibroblasts in the GTEx data. Using DRAGON, we also generated an aggregate miRNA network from the 938 CCLE cell lines that had both miRNA and gene expression measurements. Finally, we generated 1376 single-sample TF networks with LIONESS using CCLE gene expression data from the 1376 cell lines that had gene expression data corresponding to 35 cancer types.

\section{ANALYSIS TOOLS IN GRAND}

Finding small molecule candidates through reverse gene targeting

The hypothesis underlying our GRN analysis is that changes in the targeting of genes by TFs represents regulatory differences that underlie phenotypic diversity, including the potential to respond to particular stimuli. These analyses generally search for differentially targeted genes 

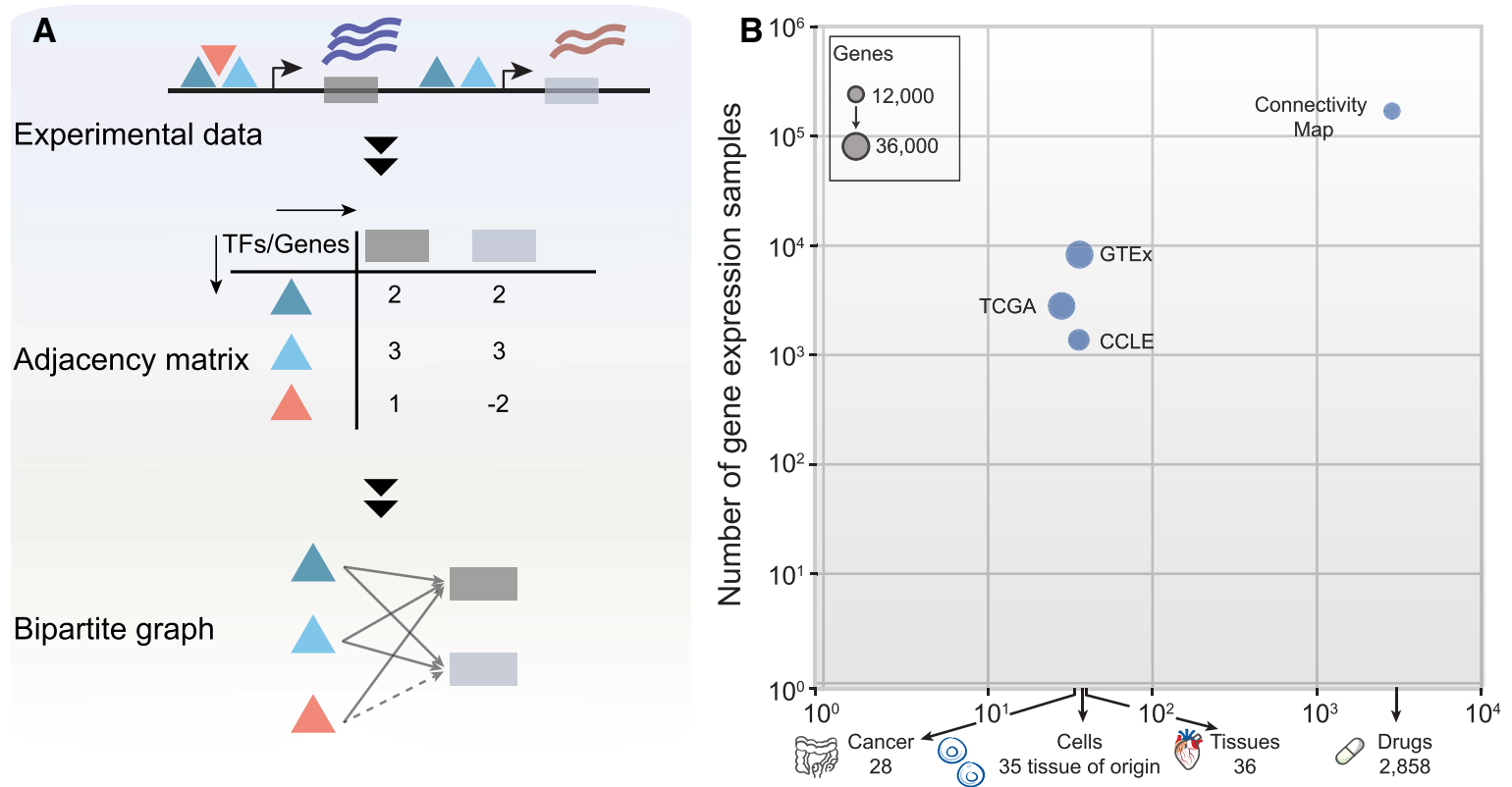

C Protein-protein interaction
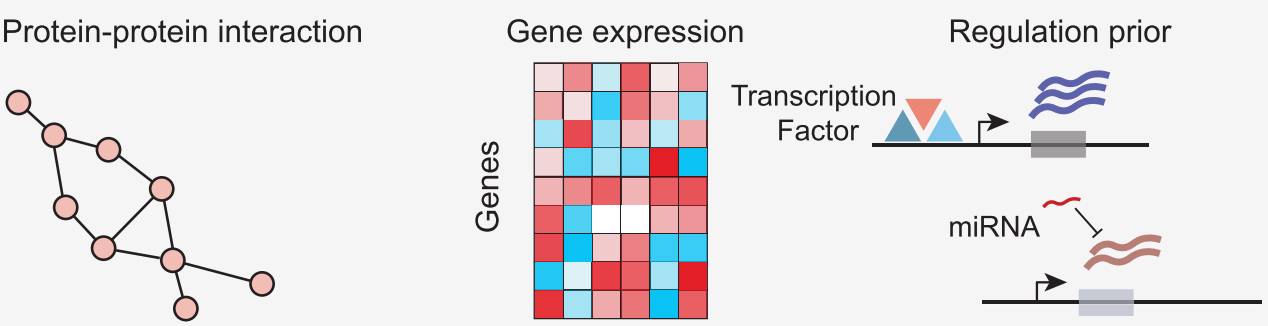

Experimental data
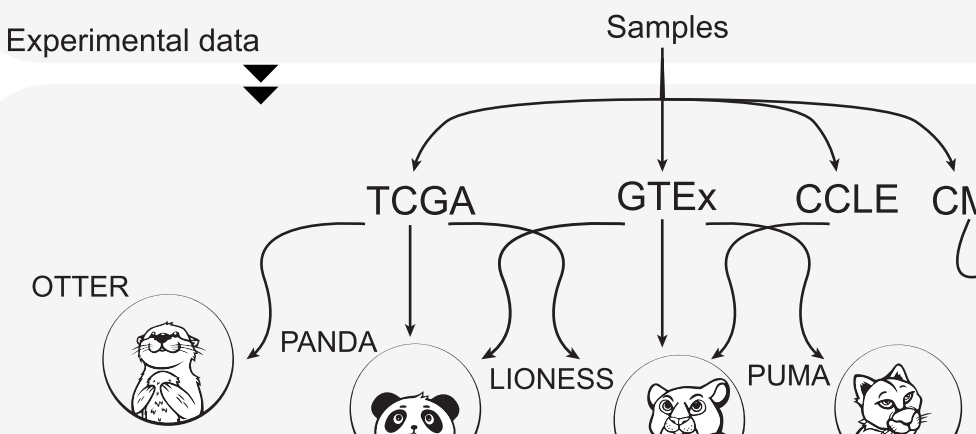

Network inference


The Network

Zoo

netzoo.github.io

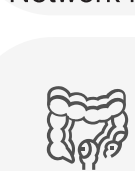

Cancer resource

28 cancer types

Regulator: TF

2,811 patient-specific networks

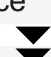

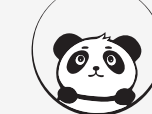

LIONESS
Tissues resource

36 tissues

Regulator: TF + miRNA

8,279 sample-specific networks
(๑)

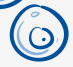

Cell line resource 37 tissue of origin

Regulator: TF + miRNA

1,378 cell line-specific networks

Public database (GRAND)

Figure 1. GRAND database statistics and network reconstruction pipeline. (A) Regulators (TFs) bind in the promoter region of target genes and affect their expression, which can be represented as a bipartite graph and its adjacency matrix. (B) Representation of the largest gene expression datasets in each of the GRAND resources. X-axis indicates the number of cancer types, tissues types, cell line tissues of origin, and drugs in each dataset. Y-axis indicates the number of samples used to build the networks. The bubble size is scaled by the number of genes in the networks. (C) GRNs were inferred from experimental data priors such as protein-protein interaction, gene expression and regulatory prior build from TF motifs or miRNAs predicted targets. The network inference methods that were used are available at https://netzoo.github.io/. 
A

Network view: Transcription factor

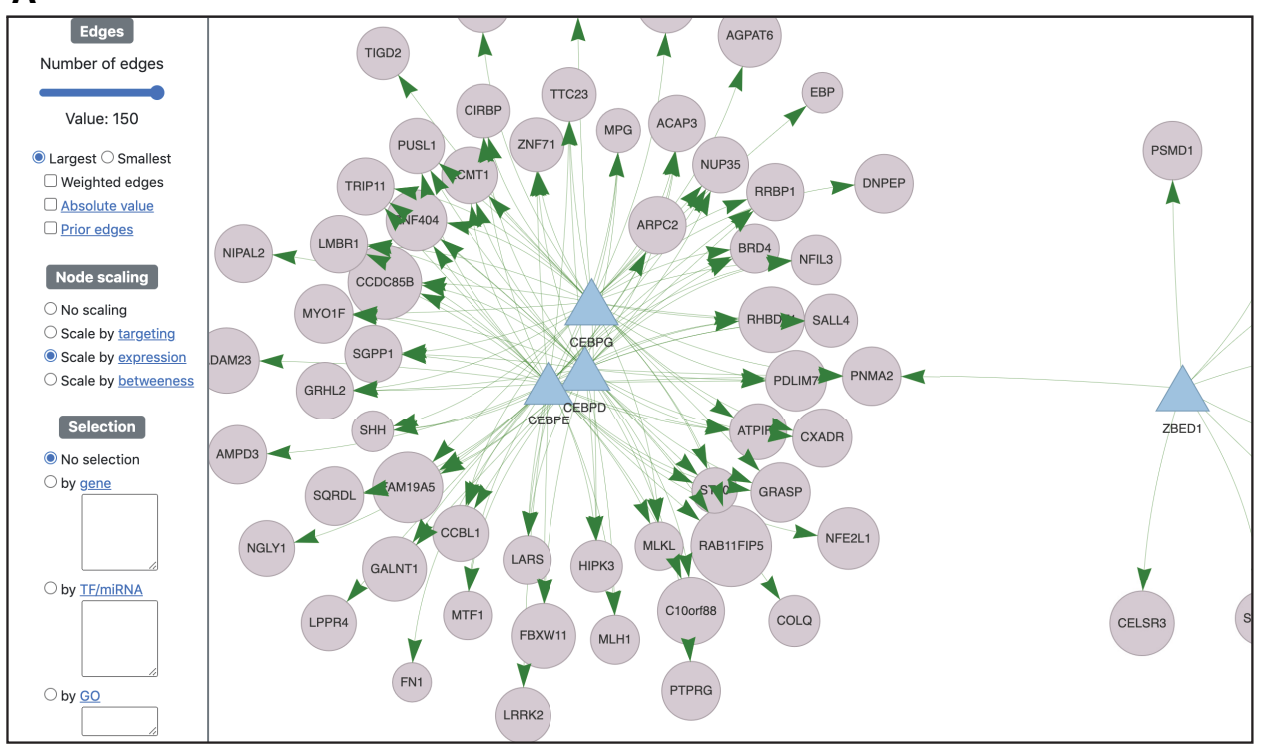

B

Network view: miRNA

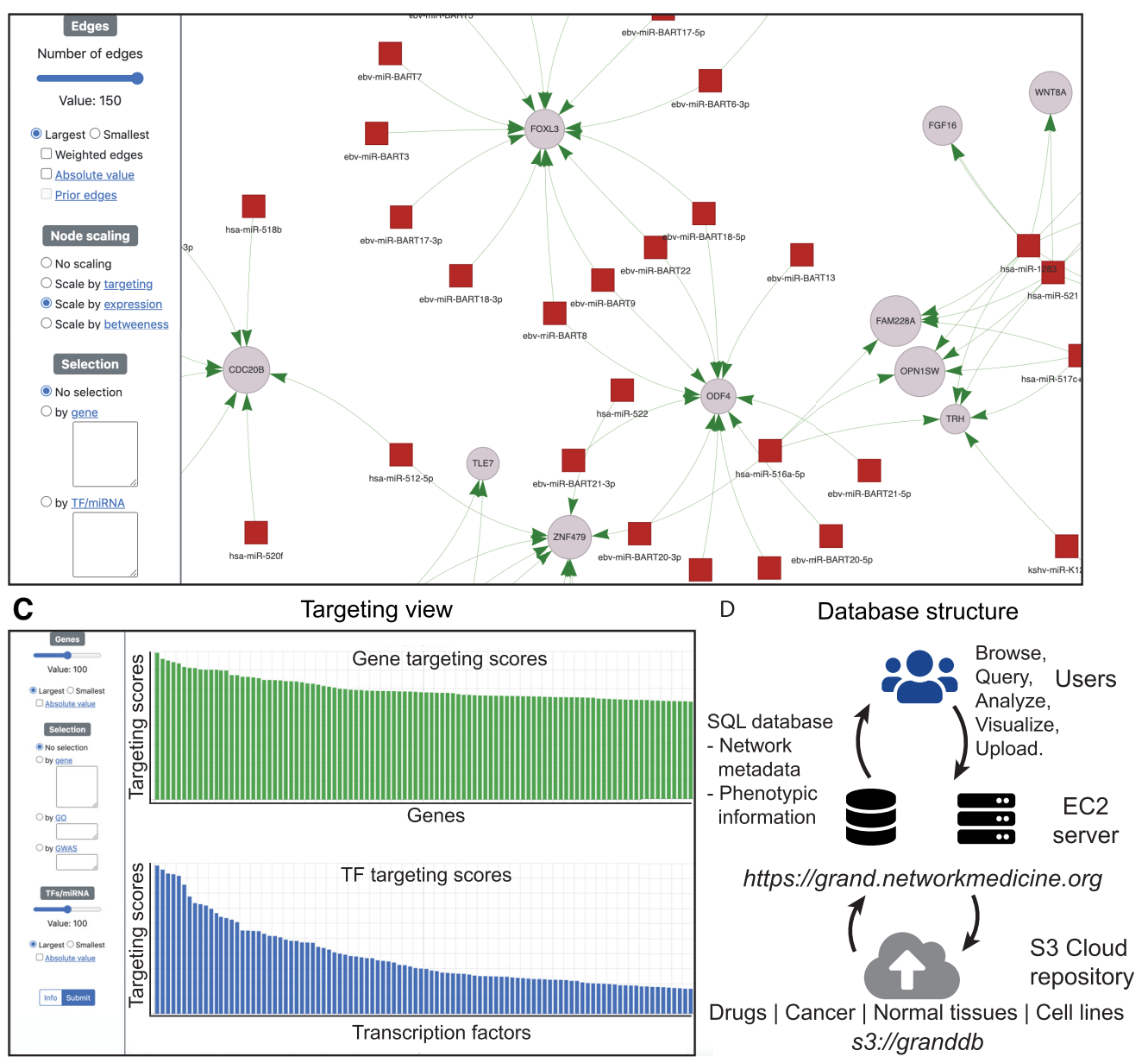

Figure 2. Gene regulatory network visualization and analysis in GRAND. Any network in GRAND can be visualized; shown in this figure are a TF GRN (A) and a miRNA GRN (B). Users can select a subset of the network using several parameters related to the edges or the nodes, such as regulators and gene sets, GO terms, and GWAS traits. Nodes can be scaled by expression, targeting or betweenness. (C) The targeting analysis allows users to calculate and visualize each network's TF and gene targeting score, and contains links to GRAND's downstream analysis tools such as functional enrichment analysis and drug repurposing. (D) Database design and infrastructure. 
or differential targeting by TFs and use functional enrichment analysis to explore functional differences between the biological states that are compared. In GRAND, we implemented a method, CLUEreg, to extend this framework to the identification of drugs that can potentially reverse disease phenotypes by allowing users to search for regulatory changes induced by small molecule compounds and other drugs profiled in the Connectivity Map (Supplementary methods).

\section{TF enrichment analysis tool}

Comparative gene regulatory network analysis generally identifies 'differential targeting' TFs that regulate different sets of genes in the phenotypes being compared. To help characterize sets of TFs, GRAND implements a hypergeometric test to compare a user-supplied list of TFs to a variety of resources, including a list of tissue-specific differential targeting and differentially expressed TFs (41), a library of 170 GWAS traits in which a GWAS SNP maps to a TF's corresponding gene (6), and a collection of TFs identified by the Human Phenotype Ontology (48) library that includes 2440 human conditions and phenotypes. The tool computes the $P$-value and the multiple testing corrected $q$-value to assess the significance of the enrichment of the term in the input TF query in the background of 1639 TFs encoded in the genome (Figure 3).

\section{DATABASE CONSTRUCTION AND USER INTERFACE}

\section{Database structure, design and implementation}

The GRAND frontend was developed in Bootstrap (v 5.0) and jQuery (v 3.3.1). Network visualization was implemented in Vis.js (v 8.5.2). Bar plots, scatter charts, and bubble plots were implemented using Chart.js (v 2.9.4) and Highcharts.js ( $\mathrm{v}$ 8.2.2). The backend was developed in Django (v 3.0.5) (49) and Python (v 3.8) (50) and deployed on a Ubuntu (v 18.04) Amazon Web Services (AWS) EC2 instance using Nginx (51) web server and SQLite (v 3.31.1) database tool which is integrated in Django (Figure 2D). Using Django for constructing the website was motivated by its versatility as it integrates a frontend tool, a database management system, and a backend tool, which provides great ease-of-use.

GRAND contains more than 6TB of network data which is hosted on a public AWS S3 bucket (s3://granddb). Although websites such as NDEx (28) allow users to host and visualize networks for up to $10 \mathrm{~GB}$ of data, the size and complexity of data in GRAND required a tailored design approach to efficiently process queries on genomescale networks with millions of edges. Finally, programmatic access to the website through the API was implemented using Django REST Framework (v 3.11). The website repository is version-controlled at https://github.com/ QuackenbushLab/grand.

\section{User interface: network browsing}

GRAND's interface was designed to allow users to browse, download, visualize and analyze the collected set of networks. The networks are organized by source type and include links from the homepage to browsable sets of network models from 'Small molecules,' 'Cancer,' 'Tissues' and 'Cell lines'; these pages can also be reached using the 'Networks' menu item in the upper right menu bar. Each page contains multiple links to brief 'help' messages that explain various fields. Clicking on one of these collections takes the user to a subpage where the subsets of the main classes can be selected. Drug targeting scores are classified by the drug name, with an interactive bubble plot that provides information about the differentially targeted TFs and genes as well as the number of samples in each drug. The 'Cancer' page classifies cancer types by tissue of origin. Three bar plots summarize the number of samples, TFs, and genes in each network and allow users to access the cancer type of interest by clicking on bars within the plots (Supplementary Figure S1A). The 'Tissues' page lists all 36 tissues in a data table. A bar plot summarizes the number of sample-specific networks available in each category (Supplementary Figure S1A). A second bar plot categorizes networks by regulation modality (TF or miRNA). These plots are interactive and clicking on individual bars filters the table below. The 'Cell lines' page contains networks categorized into three sets: cancer cell line networks from CCLE, normal cell line networks from GTEx, and a miRNA aggregate network. Cancer cell lines are grouped by cancer type and an interactive bar plot lists the number of samples in each category (Supplementary Figure S1A). A second, interactive bubble plot shows the size (number of TFs, miRNA, genes, and sample) in each of the three sets.

Clicking on a cell line/cancer/tissue link within these summary pages leads to an individual network page that lists available networks for the given category. In addition, the page provides sortable metadata used for network inference as well as additional metadata, including basic statistics on the type and number of regulators, genes, and samples used to reconstruct the network. In the 'Cancer' and 'Tissues' sections, the sample number links to the phenotypic variables associated with each sample (Supplementary Figure S1B). In the 'Cell line' and 'Small molecules' sections, information is provided on the cell line and drug dosage as appropriate. In the 'Small molecules' page, clicking the 'Genes' column opens a table containing the gene names and their attributes. In all pages, clicking on the entry in the 'Reference' column either links to the relevant published study, or, for the 'Small molecules' page, to the relevant entry in PubChem. Each drug in the 'Small molecules' section includes a panel with information about the drug indication, its chemical structure, and several relevant parameters compiled from the DRH (46) and the Connectivity Map (24) (Supplementary Figure S2). In addition to the network information page, relevant metadata about the samples used in the analysis are available in the 'Phenotypic information' table.

The networks and associated metadata can be downloaded, either in bulk or individually, from both the web interface and the API. Users can specify whether to download the networks as either TF-by-gene adjacency matrices using the 'Adj' button or lists of TF-gene edges using the 'Edge' button. The 'Vis' button links to the integrated visualization module that allows users to produce interactive graphs of regulatory networks (see the section on network visualization below). 


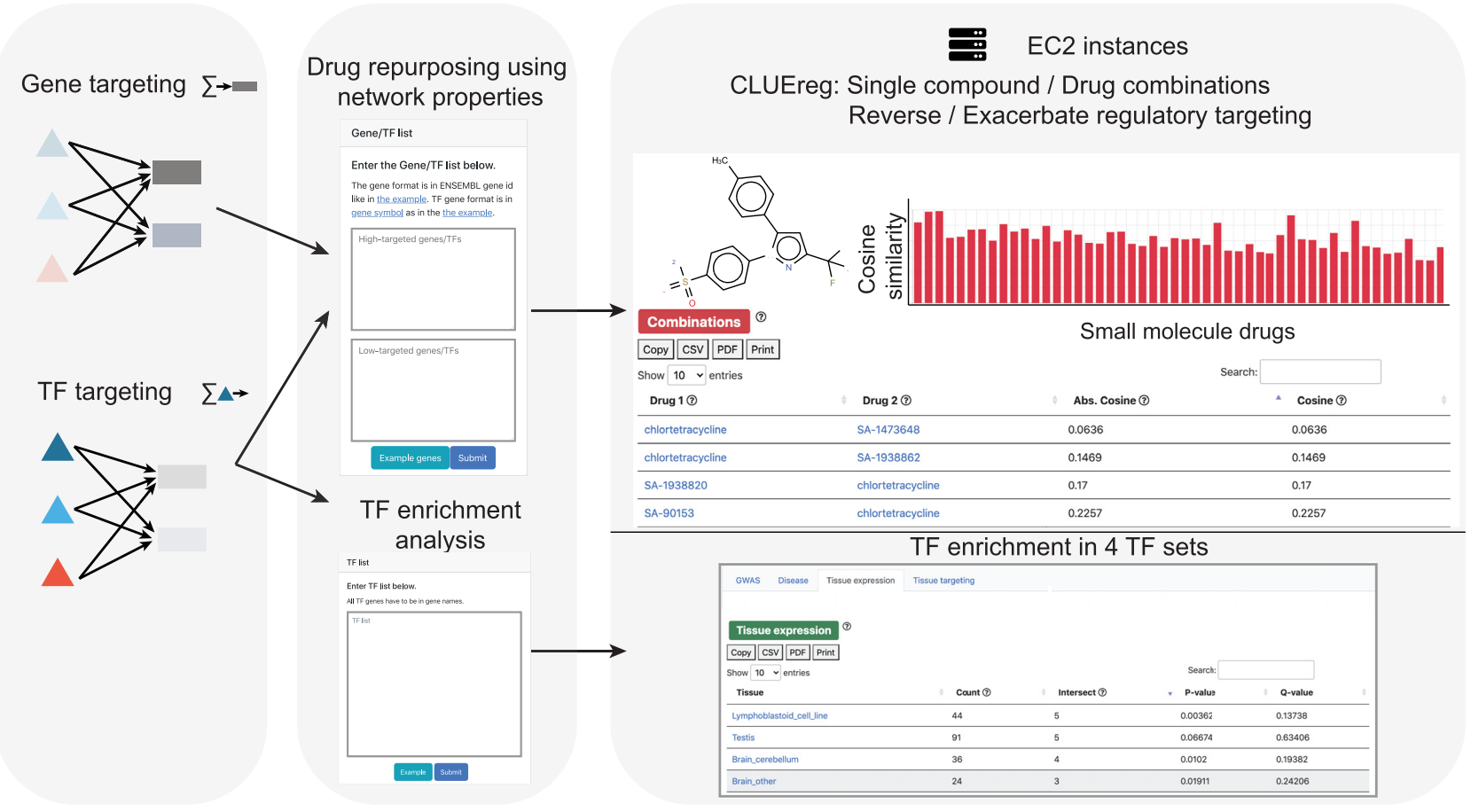

Figure 3. Analysis tools and the web server functionalities in GRAND. A list of up-targeted and down-targeted genes or TFs computed from a weighted bipartite network are given as an input to CLUEreg, which then computes similarity scores to the targeting scores of 19791 small molecules to find the single and combination candidates that reverse or exacerbate the input signature. A second feature allows users to perform an enrichment analysis of a list of TFs against four TF sets: TFs linked to disease phenotypes through GWAS or the Human Phenotype Ontology and differentially expressed or differentially targeting TFs in specific tissues.

Finally, reflecting our commitment to reproducible research, clicking on the 'Code' button in each network links to the code used to generate the networks along with information about the parameters used in the analysis. For networks generated using MATLAB, the code is provided as '.m' files, while for Python and R, Jupyter notebooks are provided that can be run through the webserver 'netbooks' (http://netbooks.networkmedicine.org).

\section{User interface: network visualization}

The network visualization tool can be accessed through the 'Vis' button in the network table and through the phenotypic variable plots. The network visualization page contains a 'network' tab and a 'targeting' tab. The 'network' tab has a selection panel that allows users to plot a TF (Figure $2 \mathrm{~A}$ ) or miRNA (Figure 2B) subnetwork using several parameters, such as the number of edges and edge weights filtered by absolute or signed values. The 'Prior' edges option plots network edges supported by the presence of a TF motif in the promoter region of target genes or miRNA target predictions. Node sizes can be scaled by the targeting score of each node, the average gene expression of the node, or the betweenness centrality of each node in the subnetwork. A regulator (TF or miRNA) and gene list submission form allows users to enter a gene or TF list of interest in both ENSEMBL gene ids and gene symbols to be selected in the network view. An additional GWAS form allows selection of genes by GWAS traits from the GWAS catalog (52). A
GO term form allows input of GO terms to select a subnetwork of the term of interest.

The 'targeting' tab (Figure 2C) computes gene and TF targeting scores in the network and allows selection based on the same parameters as in the network tab. In addition, after plotting targeting scores for the nodes of interest, an analysis section redirects the user to downstream analysis tools such as CLUEreg, for drug repurposing, or TF enrichment analysis, with prefilled forms.

\section{User interface: network analysis}

The 'Analysis' section provides access to four web server tools: CLUEreg, TF enrichment analysis, network comparison, and visualization and integrated analyses of user-provided networks (Figure 3). While CLUE (CMap and LINCS Unified Environment; https://clue.io) (24) uses gene expression to match drug perturbations to input disease gene lists, CLUEreg uses the properties of inferred regulatory networks to identify drugs that may 'correct' aberrant regulatory patterns. The CLUEreg page provides two panels allowing users to enter lists of 'high-targeted' and 'low-targeted' genes or TFs in the disease of interest. Users can query by gene symbols, ENSEMBL gene ids or mixed lists, by target genes or TFs, and by including or excluding investigatory drugs. An additional option computes optimal drug combinations. CLUEreg outputs the top small molecules that either reverse or enhance the differential targeting in disease, including summary statistics (cosine 
similarity, overlap, $P$-value, q-value, and tau-value described in Supplementary methods). Each row in the result table has an 'expand' button that shows the chemical structure and basic information about the drug. The results are also displayed as an interactive bar plot. Clicking on the plot filters the result table for the compound of interest.

The TF enrichment analysis allows users to input a set of TFs in gene symbol, ENSEMBL gene ids or mixed lists and test the enrichment against four TF sets: TFs linked to disease phenotypes through GWAS (6), TFs annotated to disease through the Human Phenotype Ontology (48), and TFs that have previously been identified as either differentially expressed or differentially targeting in specific tissues (41). The results are presented in interactive bar plots and tables showing the enrichment statistics ( $P$-value and $q$-values).

The 'Upload your own network' tab allows users to upload an adjacency matrix as a file of $500 \mathrm{Mb}$ maximum and visualizes the network using an integrated module, perform differential targeting analyses, and export the results to either CLUEreg or Enrichment analysis using pre-filled forms.

In addition to using CLUEreg and TF enrichment tools on user-provided gene lists, these tools can be used on any network in GRAND. From the visualization page of a given network, users can run these downstream analyses on a subnetwork of interest. Finally, in the 'Network comparison' tab, differential network analyses can be performed on a set of cancer and normal tissues to find regulatory disruptions involved in malignant processes. These networks were generated using the same gene expression and network inference pipeline to remove variability due to parameter choice.

\section{Additional information and API}

GRAND includes a 'Help' page that contains extensive information detailing the various sections of the website. Programmatic access is enabled through an API implemented using Django REST Framework to allow batch downloads and integration into computational pipelines. The API functions and documentation, as well as Python and MATLAB tutorials are provided in the help page.

\section{EXAMPLE ANALYSIS: COMPARING COLON CAN- CER AND NORMAL COLON NETWORKS}

To demonstrate the use of GRAND, we compared networks from modeled colon cancer and normal colon tissues to identify differentially targeted genes in cancer and to suggest small molecules that can potentially reverse the diseasespecific network perturbation. We compared an aggregate PANDA network for colon cancer (42) and the corresponding normal tissue network (41) that had been published using data from TCGA (10) and GTEx (9), respectively. We pruned each network to include only the 12817 genes and 661 TFs appearing in both.

To compare these networks, we simply subtracted the cancer network from the normal network (Figure 4A). We calculated a targeting score for the genes and TFs as the sum of the weighted in-degree or out-degree, respectively. The genes and TFs were ranked by their respective weights. The 300 genes with the highest and 300 genes with the lowest weights in the differential network were selected for analysis in GRAND; similarly, the 100 highest and the 100 lowest targeting TFs were selected (Figure 4B). We analyzed these gene and TF sets using CLUEreg.

CLUEreg identified a number of drugs as candidates likely to reverse the differentially targeted genes scores in colon cancer. The known anti-cancer compound CB7950998 was among the highest-ranked (rank 3 overall with a cosine similarity of -0.054 ); in particular CB7950998 was predicted to reverse the targeting of DCXR and MPL20 (Figure 4C), two genes known to be dysregulated in colon cancer. CB7950998 has been suggested to increase the chemosensitivity through acting as $A H R$ agonist, however with limited activity in vivo (53).

In analyzing the TF targeting scores, CLUEreg identified MK-5108 (rank 1 with a cosine similarity of -0.32) (Figure $4 \mathrm{C}$ ) as the most likely drug to reverse regulatory targeting in colon cancer and suggests that it works primarily by targeting transcription factor $F O X P 4$. MK-5108 is an investigational drug that targets aurora A kinase, a proliferation marker (54) that plays a central role in mitosis (55). Using GRAND to search for the regulatory pattern of MK-5108, we find that the drug is associated with 192 low-targeting TFs and 41 high-targeting TFs (Figure 4D). We then used these TFs as input to CLUEreg to search for compounds with similar targeting patterns. This identified PF-543, a sphingosine kinase inhibitor that alters lymphocyte trafficking (Figure 4E) (56), and Trametinib, an inhibitor of MEK1 and MEK2 that has shown promise in clinical trials for colorectal cancer (57) and metastatic melanoma (58) carrying the BRAF V600E mutation (58).

To further investigate the potential activity of MK-5108, we analyzed the functional roles of the TFs using the TF enrichment tool in GRAND. Searching the list of 233 TFs against the GWAS hits library, type 2 diabetes, breast cancer, and colorectal cancer were identified as the first, second, and seventh most significant GWAS traits (Figure 4F). The search against the Human Phenotype Ontology identified diabetes and seizures as the top traits associated with MK-5108, which may indicate that these could be possible adverse reactions associated with MK-5108. The search of the MK-5108 against the 'normal' tissue expression and tissue targeting identified an association with transverse colon tissue as well as the lymphoblast and fibroblast cell lines. The former is logical as MK-5108 is predicted to be effective against colon cancer, the latter cell lines also make sense because MK-5108 targets the mitotic process and these cell lines are known to have altered cell cycle processes relative to their tissues of origin.

While only suggestive and requiring validation experiments, the lines of evidence from multiple sources suggest that MK-5108 may be an agent with efficacy in treating colon cancer by altering regulatory patterns in the disease. More importantly, this example demonstrates the potential value of the GRAND database and its associated search tools and underscores the value of methods for gene regulatory network inference. 


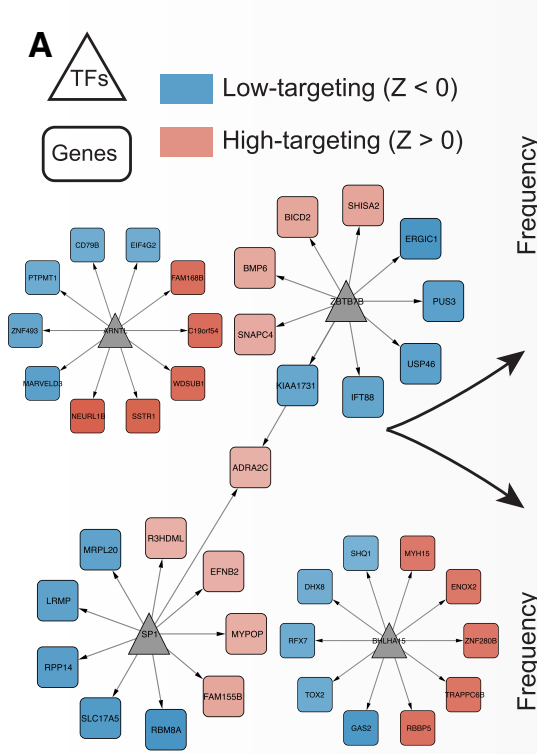

Differential colorectal cancer regulatory network
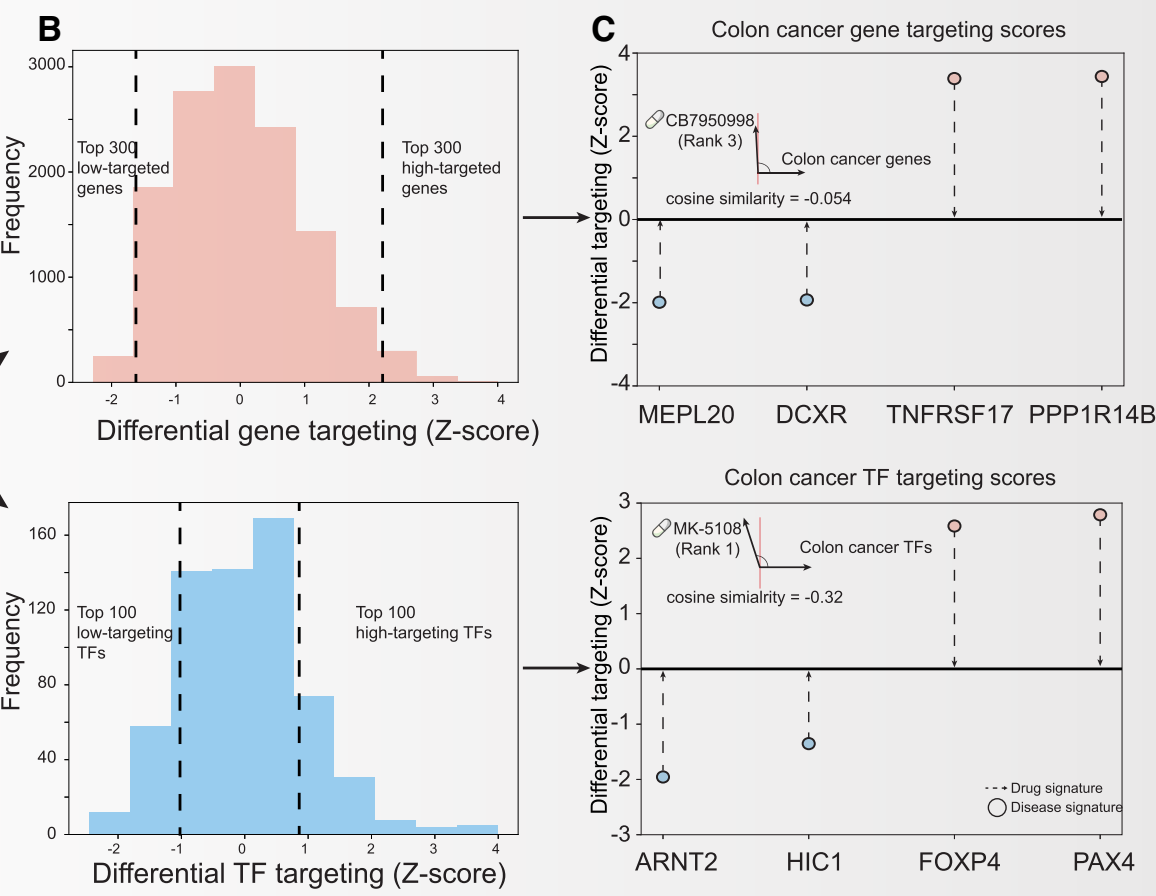

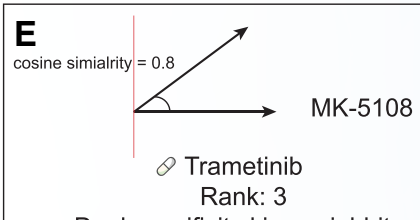

Dual specificity kinase inhbitor MEK-mediated regulation of cell growth

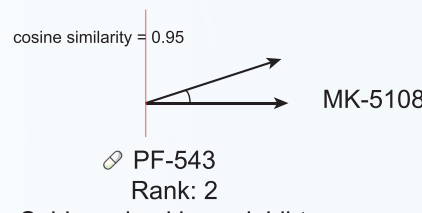

Sphingosine kinase inhibtor Key regulation of lymphocyte trafficking
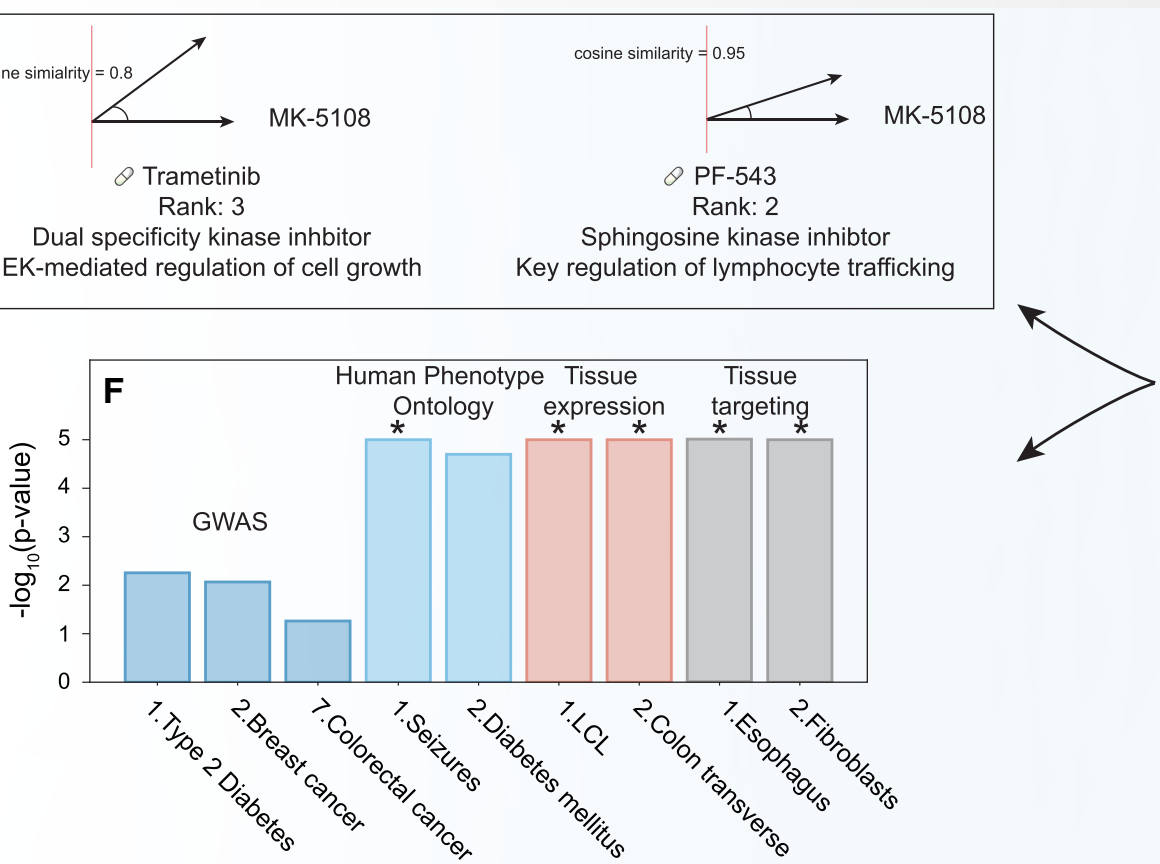

\section{MK-5108 TF targeting}

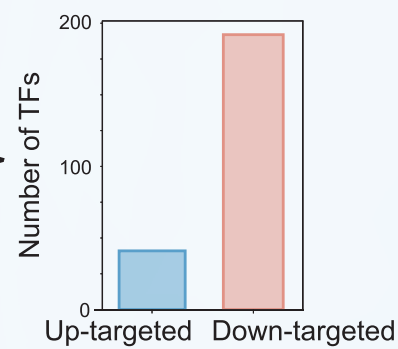

Figure 4. Integrative analysis of colon cancer network using GRAND combined tools. (A) A differential network between the colon cancer network and the normal transverse colon network allows the selection of the top differential targeted genes and the top differential targeting TFs (B). (C) CLUEreg analysis suggested two compounds MK-5108 and CB7950998 to reverse the colon cancer network targeting score. (D) The TF targeting scores of MK5108, an investigational kinase inhibitor, is similar to the scores of two other known kinase inhibitors. (E) Both kinases have different physiological roles which could set the basis for a combination therapy. (F) TF enrichment analysis of MK-5108 TF targeting scores suggested a possible specificity for colon tissue. $* P$-value $<10^{-5}$. 


\section{Conclusions and future development}

An increasing number of studies involves the inference of GRNs and their subsequent analysis. This increase is driven in part by the recognition that GRNs allow identification of biologically significant processes associated with a wide range of phenotypes that can be missed when looking at gene expression alone. Despite the utility of GRNs, published studies have generally failed to provide access to the GRNs themselves because the size of the inferred networks can exceed size limits for supplementary data allowed by journals and websites and because there have been no public repositories for these genome-scale models. Although readers of these studies could recreate the networks used in the analyses, the time and cost of inferring hundreds or thousands of large-scale networks at the sample level can be prohibitive. These difficulties with recreating the networks limit both assessment of the reproducibility of published studies and the use of the inferred GRNs for additional analyses.

GRAND represents a curated large-scale repository for genome-scale GRNs paired with extensive phenotypic information. In its current release, GRAND is populated with 12468 GRNs and 173013 targeting scores linking TFs and miRNAs to their target genes using a collection of GRN inference methods available in netzoo. Future releases of GRAND will include additional gene regulatory network models from an increasing number of biological contexts, as well as networks inferred using newly developed inference methods designed to take advantage of the ever more complex multi-omics data that we can now generate. In addition, we will include models inferred from additional public data sets, including a larger number of cancer regulatory models and GRNs inferred from single-cell expression data. We also plan to include additional analytical tools and features requested by users of the resource.

\section{DATA AVAILABILITY}

GRAND is accessible at https://grand.networkmedicine. org and all source code is available at https:/github.com/ QuackenbushLab/grand.

\section{SUPPLEMENTARY DATA}

Supplementary Data are available at NAR Online.

\section{ACKNOWLEDGEMENTS}

The authors would like to acknowledge Tian Wang and Yunhao Huo for the assistance with graphical design, Dr. Alberto Noronha and the Quackenbush lab members for helpful discussions. Icons in Figures 1 and 2 were used from Font Awesome using the following license https:// fontawesome.com/license.

\section{FUNDING}

M.L.K. is supported by grants from the Norwegian Research Council, Helse Sør-Øst and University of Oslo through the Centre for Molecular Medicine Norway
(NCMM); K.G. is supported by a grant from the National Heart, Lung, and Blood Institute, National Institutes of Health [K25 HL133599]; J.P. is supported by the National Heart, Lung and Blood Institute [K25 HL140186]; C.M.L.R., R.B., D.W., M.B.G. and J.Q. are supported by a grant from the National Cancer Institute, National Institutes of Health [R35 CA220523]; J.Q. and M.B.G. are also supported by U24 CA231846; B.S. is supported by 1T32CA236764. Funding for open access charge: National Institutes of Health [R35 CA220523].

Conflict of interest statement. None declared.

\section{REFERENCES}

1. Lee,T.I. and Young,R.A. (2013) Transcriptional regulation and its misregulation in disease. Cell, 152, 1237-1251.

2. Ando,M., Saito,Y., Xu,G., Bui,N.Q., Medetgul-Ernar,K., Pu,M., Fisch,K., Ren,S., Sakai,A. and Fukusumi,T. (2019) Chromatin dysregulation and DNA methylation at transcription start sites associated with transcriptional repression in cancers. Nat. Commun., $10,2188$.

3. Hobert,O. (2008) Gene regulation by transcription factors and microRNAs. Science, 319, 1785-1786.

4. Chen,K. and Rajewsky,N. (2007) The evolution of gene regulation by transcription factors and microRNAs. Nat. Rev. Genet., 8, 93-103.

5. O’Brien,J., Hayder,H., Zayed,Y. and Peng,C. (2018) Overview of microRNA biogenesis, mechanisms of actions, and circulation. Front. Endocrinol., 9, 402.

6. Lambert,S.A., Jolma,A., Campitelli,L.F., Das,P.K., Yin,Y., Albu,M., Chen,X., Taipale,J., Hughes,T.R. and Weirauch,M.T. (2018) The human transcription factors. Cell, 175, 598-599.

7. Chèneby,J., Ménétrier,Z., Mestdagh,M., Rosnet,T., Douida,A., Rhalloussi,W., Bergon,A., Lopez,F. and Ballester,B. (2020) ReMap 2020: a database of regulatory regions from an integrative analysis of human and Arabidopsis DNA-binding sequencing experiments. Nucleic Acids Res., 48, D180-D188.

8. Zentner,G.E., Kasinathan,S., Xin,B., Rohs,R. and Henikoff,S. (2015) ChEC-seq kinetics discriminates transcription factor binding sites by DNA sequence and shape in vivo. Nat. Commun., 6, 8733.

9. Lonsdale,J., Thomas,J., Salvatore,M., Phillips,R., Lo,E., Shad,S., Hasz,R., Walters,G., Garcia,F. and Young,N. (2013) The genotype-tissue expression (GTEx) project. Nat. Genet., 45, 580-585.

10. Weinstein,J.N., Collisson,E.A., Mills,G.B., Shaw,K.R.M., Ozenberger,B.A., Ellrott,K., Shmulevich,I., Sander,C. and Stuart,J.M. (2013) The cancer genome atlas pan-cancer analysis project. Nat. Genet., 45, 1113-1120.

11. Irrthum,A., Wehenkel,L. and Geurts,P. (2010) Inferring regulatory networks from expression data using tree-based methods. PLoS One, 5, e12776.

12. Margolin,A.A., Nemenman,I., Basso,K., Wiggins,C., Stolovitzky,G., Dalla Favera,R. and Califano,A. (2006) ARACNE: an algorithm for the reconstruction of gene regulatory networks in a mammalian cellular context. BMC Bioinformatics, 7, S7.

13. Haury,A.-C., Mordelet,F., Vera-Licona,P. and Vert,J.-P. (2012) TIGRESS: trustful inference of gene regulation using stability selection. BMC Syst. Biol., 6, 145.

14. Lee,S., Zhang,C., Kilicarslan,M., Piening,B.D., Bjornson,E., Hallström,B.M., Groen,A.K., Ferrannini,E., Laakso,M. and Snyder,M. (2016) Integrated network analysis reveals an association between plasma mannose levels and insulin resistance. Cell Metab., 24, 172-184

15. Reiss,D.J., Plaisier,C.L., Wu,W.-J. and Baliga,N.S. (2015) cMonkey2: automated, systematic, integrated detection of co-regulated gene modules for any organism. Nucleic Acids Res., 43, e87.

16. Nicolle,R., Radvanyi,F. and Elati,M. (2015) CoRegNet: reconstruction and integrated analysis of co-regulatory networks. Bioinformatics, 31, 3066-3068.

17. Aibar,S., González-Blas,C.B., Moerman,T., Imrichova,H., Hulselmans,G., Rambow,F., Marine,J.-C., Geurts,P., Aerts,J. and van 
den Oord,J. (2017) SCENIC: single-cell regulatory network inference and clustering. Nat. Methods, 14, 1083-1086.

18. Specht,A.T. and Li,J. (2017) LEAP: constructing gene co-expression networks for single-cell RNA-sequencing data using pseudotime ordering. Bioinformatics, 33, 764-766.

19. Matsumoto,H., Kiryu,H., Furusawa,C., Ko,M.S., Ko,S.B., Gouda,N., Hayashi,T. and Nikaido,I. (2017) SCODE: an efficient regulatory network inference algorithm from single-cell RNA-Seq during differentiation. Bioinformatics, 33, 2314-2321.

20. Papili Gao,N., Ud-Dean,S.M., Gandrillon,O. and Gunawan,R. (2018) SINCERITIES: inferring gene regulatory networks from time-stamped single cell transcriptional expression profiles. Bioinformatics, 34, 258-266.

21. Sanchez-Castillo,M., Blanco,D., Tienda-Luna,I.M., Carrion,M. and Huang, Y. (2018) A Bayesian framework for the inference of gene regulatory networks from time and pseudo-time series data. Bioinformatics, 34, 964-970.

22. Woodhouse,S., Piterman,N., Wintersteiger,C.M., Göttgens,B. and Fisher,J. (2018) SCNS: a graphical tool for reconstructing executable regulatory networks from single-cell genomic data. BMC Syst. Biol., 12,59

23. Song,Q., Lee,J., Akter,S., Rogers,M., Grene,R. and Li,S. (2020) Prediction of condition-specific regulatory genes using machine learning. Nucleic Acids Res., 48, e62.

24. Subramanian,A., Narayan,R., Corsello,S.M., Peck,D.D., Natoli,T.E., Lu,X., Gould,J., Davis,J.F., Tubelli,A.A. and Asiedu,J.K. (2017) A next generation connectivity map: L1000 platform and the first 1,000,000 profiles. Cell, 171, 1437-1452.

25. Duran-Frigola,M., Pauls,E., Guitart-Pla,O., Bertoni,M., Alcalde,V., Amat,D., Juan-Blanco,T. and Aloy,P. (2020) Extending the small-molecule similarity principle to all levels of biology with the chemical checker. Nat. Biotechnol., 38, 1087-1096.

26. Keenan,A.B., Jenkins,S.L., Jagodnik,K.M., Koplev,S., He,E., Torre,D., Wang,Z., Dohlman,A.B., Silverstein,M.C. and Lachmann,A. (2018) The library of integrated network-based cellular signatures NIH program: system-level cataloging of human cells response to perturbations. Cell Syst., 6, 13-24.

27. Fang,L., Li,Y., Ma,L., Xu,Q., Tan,F. and Chen,G. (2021) GRNdb: decoding the gene regulatory networks in diverse human and mouse conditions. Nucleic Acids Res, 49, D97-D103.

28. Pratt,D., Chen,J., Welker,D., Rivas,R., Pillich,R., Rynkov,V., Ono,K., Miello,C., Hicks,L. and Szalma,S. (2015) NDEx, the network data exchange. Cell Syst., 1, 302-305.

29. Arif,M., Zhang,C., Li,X., Güngör,C., Cakmak,B., Arslantürk,M., Tebani,A., Özcan,B., Subaş,O. and Zhou,W. (2021) iNetModels 2.0: an interactive visualization and database of multi-omics data. bioRxiv doi: https://doi.org/10.1101/662502, 14 January 2021, preprint: not peer reviewed.

30. Wong,A.K., Krishnan,A. and Troyanskaya,O.G. (2018) GIANT 2.0: genome-scale integrated analysis of gene networks in tissues. Nucleic Acids Res., 46, W65-W70.

31. Arif,M., Zhang,C., Li,X., Gungor,C., Cakmak,B., Arslanturk,M., Tebani,A., Ozcan,B., Subas,O., Zhou,W. et al. (2021) iNetModels 2.0: an interactive visualization and database of multi-omics data. Nucleic Acids Res., 49, W271-W276.

32. Keenan,A.B., Torre,D., Lachmann,A., Leong,A.K., Wojciechowicz,M.L., Utti,V., Jagodnik,K.M., Kropiwnicki,E., Wang,Z. and Ma'ayan,A. (2019) ChEA3: transcription factor enrichment analysis by orthogonal omics integration. Nucleic Acids Res., 47, W212-W224.

33. Agarwal,V., Bell,G.W., Nam,J.-W. and Bartel,D.P. (2015) Predicting effective microRNA target sites in mammalian mRNAs. elife, $\mathbf{4}$ $\mathrm{e} 05005$.

34. Ghandi,M., Huang,F.W., Jané-Valbuena,J., Kryukov,G.V., Lo,C.C., McDonald,E.R., Barretina,J., Gelfand,E.T., Bielski,C.M. and Li,H (2019) Next-generation characterization of the cancer cell line encyclopedia. Nature, 569, 503-508.

35. Glass,K., Huttenhower,C., Quackenbush,J. and Yuan,G.C. (2013) Passing messages between biological networks to refine predicted interactions. PLoS One, $\mathbf{8}$, e64832.

36. Kuijjer,M.L., Fagny,M., Marin,A., Quackenbush,J. and Glass,K. (2020) PUMA: PANDA using microRNA associations. Bioinformatics, 36, 4765-4773.
37. Kuijjer,M.L., Tung,M.G., Yuan,G., Quackenbush,J. and Glass,K. (2019) Estimating sample-specific regulatory networks. Iscience, $\mathbf{1 4}$ 226-240.

38. Weighill,D., Guebila,M.B., Lopes-Ramos,C., Glass,K., Quackenbush,J., Platig,J. and Burkholz,R. (2020) Gene regulatory network inference as relaxed graph matching. bioRxiv doi: https://doi.org/10.1101/2020.06.23.167999, 24 June 2020, preprint: not peer reviewed.

39. Weighill,D., Burkholz,R., Guebila,M.B., Zacharias,H.U., Quackenbush,J. and Altenbuchinger,M. (2021) DRAGON determining regulatory associations using graphical models on multi-omic networks. arXiv doi: https://arxiv.org/abs/2104.01690, 04 April 2021, preprint: not peer reviewed.

40. Lopes-Ramos,C.M., Paulson,J.N., Chen,C.-Y., Kuijjer,M.L., Fagny,M., Platig,J., Sonawane,A.R., DeMeo,D.L., Quackenbush,J. and Glass,K. (2017) Regulatory network changes between cell lines and their tissues of origin. BMC Genomics, 18, 723.

41. Sonawane,A.R., Platig,J., Fagny,M., Chen,C.-Y., Paulson,J.N., Lopes-Ramos,C.M., DeMeo,D.L., Quackenbush,J., Glass,K. and Kuijjer,M.L. (2017) Understanding tissue-specific gene regulation. Cell Rep., 21, 1077-1088.

42. Lopes-Ramos,C.M., Kuijjer,M.L., Ogino,S., Fuchs,C.S., DeMeo,D.L., Glass,K. and Quackenbush,J. (2018) Gene regulatory network analysis identifies sex-linked differences in colon cancer drug metabolism. Cancer Res. , 78, 5538-5547.

43. Lopes-Ramos,C.M., Belova,T., Brunner,T., Quackenbush,J. and Kuijjer,M.L. (2021) Regulation of PD1 signaling is associated with prognosis in glioblastoma multiforme. bioRxiv doi: https://doi.org/10.1101/2021.02.11.430786, 12 February 2021, preprint: not peer reviewed

44. Weighill,D., Ben Guebila,M., Glass,K., Platig,J., Yeh,J.J. and Quackenbush,J. (2021) Gene targeting in disease networks. Front. Genet, 12, 501-507.

45. Lopes-Ramos,C.M., Chen,C.-Y., Kuijjer,M.L., Paulson,J.N., Sonawane,A.R., Fagny,M., Platig,J., Glass,K., Quackenbush,J. and DeMeo,D.L. (2020) Sex differences in gene expression and regulatory networks across 29 human tissues. Cell Rep., 31, 107795.

46. Corsello,S.M., Bittker,J.A., Liu,Z., Gould,J., McCarren,P., Hirschman,J.E., Johnston,S.E., Vrcic,A., Wong,B. and Khan,M. (2017) The drug repurposing hub: a next-generation drug library and information resource. Nat. Med., 23, 405-408.

47. Guebila,M.B., Morgan,D.C., Glass,K., Kuijjer,M.L., DeMeo,D.L. and Quackenbush,J. (2021) gpuZoo: cost-effective estimation of gene regulatory networks using the graphics processing unit. bioRxiv doi: https://doi.org/10.1101/2021.07.13.452214, 14 July 2021, preprint: not peer reviewed.

48. Köhler,S., Carmody,L., Vasilevsky,N., Jacobsen,J.O.B., Danis,D., Gourdine,J.-P., Gargano,M., Harris,N.L., Matentzoglu,N. and McMurry,J.A. (2019) Expansion of the Human Phenotype Ontology (HPO) knowledge base and resources. Nucleic Acids Res. , 47, D1018-D1027.

49. Team,D.C. and Foundation,D. (2016) In: Django Software Foundation. Lawrence, Kansas.

50. Van Rossum,G. and Drake,F.L. Jr (1995) In: Python Tutorial. Centrum voor Wiskunde en Informatica Amsterdam.

51. Soni,R. (2016) In: Nginx. Springer.

52. MacArthur,J., Bowler,E., Cerezo,M., Gil,L., Hall,P., Hastings,E., Junkins,H., McMahon,A., Milano,A. and Morales,J. (2017) The new NHGRI-EBI catalog of published genome-wide association studies (GWAS Catalog). Nucleic Acids Res., 45, D896-D901.

53. Amin,M., Minton,S.E., LoRusso,P.M., Krishnamurthi,S.S., Pickett,C.A., Lunceford,J., Hille,D., Mauro,D., Stein,M.N. and Wang-Gillam,A. (2016) A phase I study of MK-5108, an oral aurora a kinase inhibitor, administered both as monotherapy and in combination with docetaxel, in patients with advanced or refractory solid tumors. Invest. New Drugs, 34, 84-95.

54. Haibe-Kains,B., Desmedt,C., Loi,S., Culhane,A.C., Bontempi,G., Quackenbush,J. and Sotiriou,C. (2012) A three-gene model to robustly identify breast cancer molecular subtypes. J. Natl. Cancer Inst., 104, 311-325.

55. Nikonova,A.S., Astsaturov,I., Serebriiskii,I.G., Dunbrack,R.L. and Golemis,E.A. (2013) Aurora A kinase (AURKA) in normal and pathological cell division. Cell. Mol. Life Sci., 70, 661-687. 
56. Schnute,ME., McReynolds,MD., Kasten,T., Yates,M., Jerome,G., Rains,JW., Hall,T., Chrencik,J., Kraus,M., Cronin,C.N. et al. (2012) Modulation of cellular S1P levels with a novel, potent and specific inhibitor of sphingosine kinase-1. Biochem. J., 444, 79-88.

57. Corcoran,R.B., André,T., Atreya,C.E., Schellens,J.H., Yoshino,T., Bendell,J.C., Hollebecque,A., McRee,A.J., Siena,S. and Middleton,G. (2018) Combined BRAF, EGFR, and MEK inhibition in patients with BRAFV600E-mutant colorectal cancer. Cancer Discov., 8 , 428-443.
58. Robert,C., Flaherty,K.T., Hersey,P., Nathan,P.D., Garbe,C., Milhem,M.M., Demidov,L.V., Hassel,J.C., Rutkowski,P., Mohr,P. et al. (2012) METRIC phase III study: Efficacy of trametinib (T), a potent and selective MEK inhibitor (MEKi), in progression-free survival (PFS) and overall survival (OS), compared with chemotherapy (C) in patients (pts) with BRAFV600E/K mutant advanced or metastatic melanoma (MM). J. Clin. Oncol., 30, LBA8509. 